\title{
IMPACTO DEL USO DE TECNOLOGÍAAUTOMATIZADA EN LA PRODUCTIVIDAD DE CIPROFLOXACINO 500 MG TABLETAS RECUBIERTAS
}

\author{
Use of automated technology and its impact on productivity ciprofloxacin $500 \mathrm{mg}$ coated tablets \\ Frank A Pérez ${ }^{1}$, José R. Juárez² \\ ${ }^{1}$ Unidad de Aseguramiento de la Calidad Laboratorio Hersil, ${ }^{2}$ Instituto de Investigación en Ciencias Farmacéuticas y Recursos \\ Naturales "Juan de Dios Guevara", Facultad de Farmacia y Bioquímica, Universidad Nacional Mayor de San Marcos
}

\section{RESUMEN}

Se evaluó el impacto del uso de tecnología automatizada en las operaciones unitarias y en la productividad de la fabricación de ciprofloxacino $500 \mathrm{mg}$ tabletas recubiertas. Se trasladaron las etapas de amasado y secado del granulado a equipos automatizados, identificando los parámetros críticos del nuevo proceso; asimismo, se cuantificaron y compararon las horas/hombre empleadas en el nuevo proceso. El proceso se llevó a cabo a través de dos etapas: amasado en amasador High Shear y secado en secador de lecho fluido. Los resultados físicos, fisicoquímicos y microbiológicos del producto obtenido por el nuevo diseño estuvieron conformes a las especificaciones de calidad previamente establecidas, como $98,67 \%$ de disolución y cumplimiento de los estudios de estabilidad acelerada. Se concluyó que el empleo de tecnología automatizada bajo el diseño propuesto fue satisfactorio, se mejoraron los atributos de calidad y se reportó un ahorro significativo de $50 \%$ aproximadamente en tiempos_horas/hombre y horas/máquina.

Palabras clave: tecnología automatizada, amasador High Shear, secador de lecho fluido, productividad.

\section{SUMMARY}

In this research was evaluated the impact of use of automated technology in unit operations and manufacturing productivity of ciprofloxacin $500 \mathrm{mg}$ coated tablets, kneading_and drying stages of granulated were moved to automated equipment, identifying critical parameters of new process; also were quantified and compared the man/hours used in new process. The process was carried out through two stages: kneading in High Shear mixer granulator and drying in fluid bed dryer. The physical, chemical and microbiological product results obtained by new design were in accordance with previously established quality specifications for this product, as $98,67 \%$ dissolution and compliance of accelerated stability studies. Were concluded that the use of automated technology under the proposed design was satisfactory, quality attributes were improved and reported a 50\% saving in time about man/hours and machine/hours.

Keywords: automated technology, High Shear mixer granulator, fluid bed dryer, productivity.

\section{INTRODUCCIÓN}

E l uso de tecnologías automatizadas en el campo de la industria, ha permitidoalcanzar estándares más altos de calidad y productividad de bienes manufacturados en muchos campos, como: farmacéutico, alimentario, metalúrgico, minero y químico ${ }^{(1)}$. Este fenómeno de innovación también tiene gran importancia en el sector de la industria farmacéutica, la cual se encarga de diseñar, elaborary distribuir medicamentos, que tienen como objetivo mejorar la calidad de vida de los pacientes y prevenir enfermedades. Los medicamentos deben ser seguros, eficaces y de calidad para el consumidor final, además de cumplir con todas las exigencias regulatorias relacionadas a los atributos y características para los que fueron fabricados ${ }^{(2-5)}$.

El reto que tiene la industria actualmente es producir medicamentos de calidad a un menor costo y en el menor tiempo posible, integrando lo que son los conceptos de calidad y productividad; esto implica la optimización de procesos, capacitación y utilización de tecnología ${ }^{(3)}$.

Existen diversos tipos de formas farmacéuticas, siendo las tabletas aquellas de mayor escala a nivel de producción en la industria, ya que desde este punto de vista, su fabricación resulta sencilla y rentable gracias a los procesos mecanizados. La producción de tabletas se inicia normalmente con la elaboración de un granulado, siendo el procedimiento más utilizado la granulación húmeda ${ }^{(4)}$.

En vista del impacto que tienen las formas farmacéuticas sólidas orales, tanto en demanda de medicamentos de calidad como en la productividad de los procesos de manufactura involucrados, se hace necesario el uso de tecnología automatizada que nos permita controlar mejor los procesos, brindando productos con el nivel de calidad adecuado, optimizando los tiempos y recursos en los procesos de una planta farmacéutica. 
Tabla 1. Resultados de humedad del granulado.

\begin{tabular}{lc}
\multicolumn{1}{c}{ Etapa } & \% Humedad \\
\hline Secador de lecho fluido $\left(1^{\circ}\right.$ parcial $)$ & 3,65 \\
Secador de lecho fluido $\left(2^{\circ}\right.$ parcial $)$ & 2,36 \\
Muestreo de todo el lote & 2,38 \\
Mezcla final & 2,17 \\
Reportado por control de calidad & 2,13 \\
\hline
\end{tabular}

Por tal motivo, el presente estudio tuvo como propósito evaluar el impacto del uso de tecnología automatizada en las operaciones unitarias y la productividad en la fabricación de ciprofloxacino 500 mg tabletas recubiertas, en contraste con el uso de tecnología convencional.

\section{MATERIALES Y MÉTODOS}

Se realizó un estudio comparativo, experimental y de corte transversal.

Para el proceso de fabricación del producto ciprofloxacino $500 \mathrm{mg}$ tabletas recubiertas, se empleó un secador de lecho fluido y un mezclador-granulador de alta velocidad, automatizados, en remplazo de los equipos convencionales amasador planetario y estufa estática. Asimismo, se utilizaron balanza analítica, balanza de humedad Mettler Toledo, cromatógrafo líquido de alta performance (HPLC), desintegrador, durómetro, friabilizador. Los reactivos de análisis fueron agua destilada, metanol HPLC, hidróxido de sodio, fosfato monobásico de potasio, hidróxido de potasio, sodio fosfato monobásico monohidratado.

La metodología empleada se basó en evaluar las diferencias entre el uso de la tecnología convencional y el empleo de la tecnología automatizada, y cuantificar el impacto positivo en la productividad. Para ello, se diseñaron las operaciones del proceso automatizado de fabricación del producto. Seevaluó esta metodología a través de datos cualitativos y cuantitativos con los cuales se verificó el cumplimiento de las especificaciones de calidad establecidas: aspecto del granulado, humedad, dosaje, disolución, uniformidad de unidades de dosificación. También se consideró la mejora
Tabla 2. Resultados de aspecto del granulado.

\begin{tabular}{cccc}
\hline \multicolumn{2}{c}{ Especificación } & Aspecto (Mezcla final) \\
\hline \multicolumn{3}{c}{ Granulado de color blanco amarillento } & Conforme \\
\hline Tabla 3. Resultados de dosaje de ciprofloxacino. \\
\hline Especificación & Muestras & $\begin{array}{c}\text { Resultado } \\
\text { (mg/803,12 mg) }\end{array}$ & $\begin{array}{c}\text { Promedio } \\
\text { (mg/803,12 mg) }\end{array}$ \\
\hline & INICIO & 528,31076 & 527,59099 \\
Ciprofloxacino & MEDIO & 526,87121 & \\
463,344mg/803,12 mg- & & 528,684896 & 528,61690 \\
566,310 mg/803,12 mg & FINAL & 542,65489 & 542,10968 \\
& & 541,56438 & \\
& & Promedio & 532,7725 \\
\hline
\end{tabular}

de productividad: ahorro de horas-hombre y horasmáquina. Las evaluaciones físicas, fisicoquímicas y microbiológicas se llevaron a cabo en cada etapa de fabricación para verificar del cumplimiento de las especificaciones establecidas y medir el impacto del uso de la tecnología automatizada empleada.

La muestra empleada para la evaluación de la conformidad de los criterios de aceptación establecidos previamente, fueron representativos del tamaño de lote.

\section{RESULTADOS Y DISCUSIÓN}

La aplicación de la nueva tecnología, en sus diferentes etapas, en cuanto a los resultados

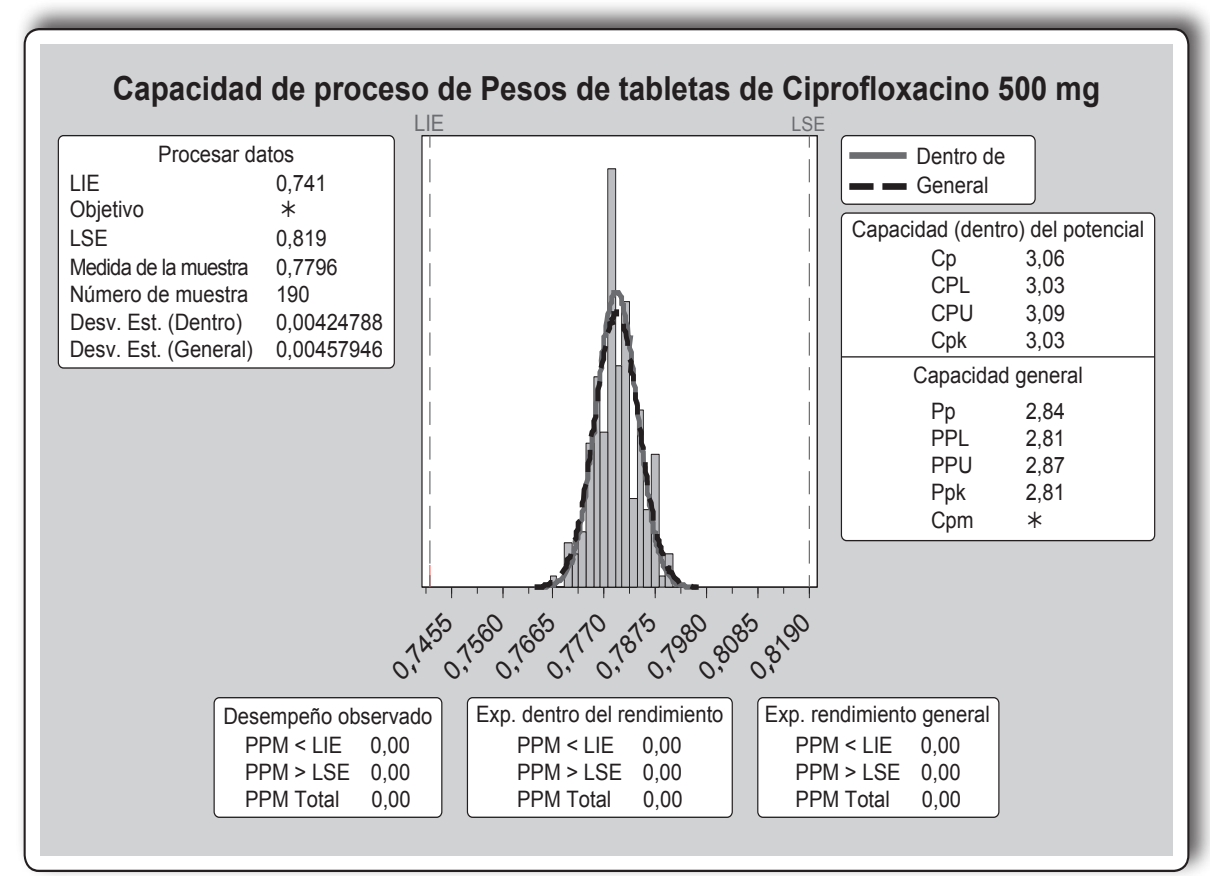

Figura 1. Evaluación de capacidad de proceso de compresión. 
obtenidos, nos muestra lo siguiente:

Etapa de granulado. En las pruebas físicas de humedad (tabla 1), aspecto (tabla 2) y granulometría, los resultados fueron similares a los productos fabricados en los equipos convencionales $y$, junto con las pruebas fisicoquímicas, cumplieron con las especificaciones establecidas. Por lo mencionado, la fabricación del producto empleando los equipos amasador High Shear y secador de lecho fluido, no afectó los atributos críticos de calidad. Sin embargo, es de resaltar que el estudio de granulometría y aspecto dieron como resultado una mejor característica granulométrica, ya que se redujo la presencia de partículas finas que pueden impactar en la etapa de compresión. Con respecto a la prueba fisicoquímica, la evaluación del dosaje en los tres niveles considerados (tabla 3) mostró homogeneidad en la mezcla. Asimismo, se obtuvo reducción (ahorro) en el nuevo método con respecto al tiempo horas-hombre y horas-máquina (tabla 4); evidenciándose un impacto positivo al aumentar la capacidad productiva de la planta por el ahorro de tiempos de proceso con el uso de nuevas tecnologías.

Etapa de compresión. El proceso fue satisfactorio debido a que se obtiene un $\mathrm{Cpk}=$ 3,03 (figura 1), lo cual indica que el proceso de compresión es capaz de producir unidades dentro de especificación, en función a las características del granulado obtenido, mediante el empleo de tecnología automatizada.

Etapa de producto terminado. Las pruebas físicas, como aspecto y peso promedio (tabla 5), y las pruebas fisicoquímicas (tabla 6), cumplieron con las especificaciones establecidas. Respecto a las pruebas físicas del producto, se encontró que los resultados conformes se correlacionan con los resultados de la etapa de granulado, en ambas etapas se cumplieron las especificaciones establecidas. En relación a las pruebas fisicoquímicas: cuantificación de principio activo, disolución y uniformidad de unidades de dosificación; los resultados estuvieron de acuerdo a las especificaciones establecidas para el producto ciprofloxacino $500 \mathrm{mg}$ tabletas recubiertas. Por lo mencionado, la fabricación del producto, empleando amasador High Shear y secador de lecho fluido, no afectaron losatributosdecalidad del productoevaluado. Adicionalmente, se pudo observar que el atributo de calidad, uniformidad de unidades de dosificación, tuvo un mejor comportamiento en contraste con los lotes realizados con tecnología convencional, siendo indicativo de mejora en la distribución del activo en la unidad de dosificación.

Finalmente, el producto fue sometido a estudios de estabilidad acelerada (condiciones: $40^{\circ} \mathrm{C}, 75 \%$ $\mathrm{HR}$ ), encontrándose conformidad en relación a los parámetros establecidos. 
Estos resultados permiten establecer que los parámetros de trabajo de los equipos amasador High Sheary secador de lecho fluido fueron adecuados y se mantuvieron constantes durante el proceso, de acuerdo al diseño establecido, para las etapas correspondientes; asimismo, los resultados del desempeño de los equipos fueron conformes.

Los parámetros de amasado y secado de los equipos fueron controlados de manera automatizada; en el caso del amasador: velocidad de la cuchilla, velocidad de la paleta, velocidad de ingreso de la solución granuladora y tiempo de amasado; en el caso del secador de lecho fluido: temperatura de aire de ingreso, temperatura del lecho de secado, velocidad de ingreso del aire y tiempo de secado.

El control de estos parámetros ayudó a obtener un granulado, en aspecto y tamaño de gránulos, con mejores características en contraste a lo realizado con el equipo convencional, amasador planetario y estufa estática, que no cuentan con control automatizado de los parámetros mencionados anteriormente.

\section{CONCLUSIONES}

- El uso de tecnología automatizada en las operaciones unitarias farmacéuticas genera un impacto positivo en la productividad, permitiendo optimizar los procesos de manufactura de productos. Ello conllevó al ahorro en las operaciones unitarias de 2 horas-hombre y horas-máquina, en promedio, por cada lote producido; esto constituye aproximadamente 50\% de ahorro en comparación con la tecnología convencional.

- El proceso de optimización de las operaciones de amasado y secado empleando nueva tecnología, amasador High Shear y secador de lecho fluido, fueron satisfactorias. Asimismo, seidentificaron los parámetros críticos del proceso de amasado y secado del producto ciprofloxacino $500 \mathrm{mg}$ tabletas recubiertas.

\section{REFERENCIAS BIBLIOGRÁFÍCAS}

1. Moreno EG. Automatización de procesos industriales. Valencia: Univ. Politec; 1999.

2. Teja SM. Automatización neumática y electroneumática. Madrid: Marcombo; 1998.

3. Vallejo B, Vallejo S. Aspectos generales de la automatización industrial del sector farmacéutico. Bogotá. Revista Colombiana de Ciencias Químico Farmacéuticas. 2006; 35(1): 47-63,

4. Salazar MR. Gestión de la calidad en el desarrolloy fabricación industrial de medicamentos. Barcelona: ROMARGRAF; 2001.

5. Gibson M. Pharmaceutical preformulation and formulation. New York: Informa Healthcare; 2009.

6. Rowe R, SheskeyP, Quinn M. Handbookof Pharmaceutical Excipients. $6^{\text {th }}$ ed. London: Pharmaceutical Press; 2009.

7. MINSA/DIGEMID. Directiva Sanitaria № 31. Directiva Sanitaria que reglamenta los estudios de estabilidad de medicamentos. Lima, 2009.

8. International Conference on Harmonization. Evaluation for stability data, ICH harmonised tripatite guideline, ICH Q1E, febrero 2003.

9. Organización Mundial de la Salud (OMS). Who guideline on transfer of technology. 2011; 285(1): 285-309.

10. USP 35 - NF 30. Cap. 3224. Rockville, MD: The United States Pharmacopeial Convention, 2012.

Manuscrito recibido el: 29/03/2016

Aceptado para su publicación el: 20/04/2016

\section{Correspondencia}

Nombre: $\quad$ Frank A. Pérez

Dirección: Av. Fruales 220 Ate - Lima

e-mail: fperezsaldana@gmail.com 